\title{
Ultrasonic-assisted Extraction and Antibacterial Activity of Polysaccharide from Lycopus lucidus Turcz
}

\author{
Shenglang Jin* \\ College of Tourism, Huangshan University, Huangshan 245021, China \\ jinshenglang@163.com \\ * Corresponding Author
}

\begin{abstract}
The response surface methodology optimizes in the study. The ultrasonic extraction process of polysaccharide from Lycopus lucidus Turcz. was optimized by using response surface methodology. The vitro antibacterial activity of roughly purified L. Lucidus polysaccharide on Escherichia coli, Bacillus subtilis, Bacillus licheniformis and Staphylococcus aureus. The optimal extraction conditions were deternaned as followed: liquid-solid ratio: $40.73 \mathrm{~mL} / \mathrm{g}$; ultrasonic power: $542.70 \mathrm{~W}$; under the optimized extraction conditions, the extraction yield of L. lucidus polysaccharide yas $8.59 \%$ (close to the theoretical value of $8.55 \%$ ). In addition, L lucidus polysaccharide showed very strong antibacterial activities E. coli, S. aureus and quite significant antibacterial activities on B. licheniformis.

Keywords: Lultrasonic wave, response surface, Ly Opus lucidus Turcz, polysaccharide, antibacterial

\section{Introduction}

Lycopus lucidus Turcz is distributed in Anhui, Zhejiang, Hunan, Hubei province of China which is one of the most abundant resources of the country leaves [1]. L.lucidus has both edible and medicinal functions. The Compendium of Materia Medica records that it can clear away heat-evil and stanch bleeding as well as detoxicate and disperse swelling. Many researchers have researched the composition of its monosaccharide, porysaccharide and its derivative, the extraction of volatile oil and the analysis of the oil composition [2]. But few report on the research of extraction and optimzation of L.lucidus polysaccharide and other compositions and its antibacterial activity.

This study explored the method of extracting water soluble polysaccharide by ultrasonic-assisted, as well as optimize the technological conditions by response surface methodology, and exerted a test on the antibacterial activity of the roughly purified polysaccharide, aiming at providing reference and theoretical premise for further development and utilization of the LTP.
\end{abstract}

\section{Materials and Methods}

\subsection{Plant Material}

L.lucidus was collected at Huangshan city in 12/2012 and 07/2013 respectively and identified by Prof. Xinsheng Tang in Huangshan University. Escherichia coli, Staphylococcus aureus, Bacillus subtilis, Bacillus licheniformis, all of these spawn were provided by biology laboratory in Huangshan University. 


\subsection{Reagents and Chemicals}

Hydrocgioric acid, petroleum ether, glucose, chloroform, N-butanol, acetocaustin 、 ferrisulfas, etheranhydrous, phenol, chloroform, concentrated sulfuric acid, Nbutanol, postassium ferricyanide, salicylic acid, and other reagents were obtained from Tianjin Haiguang Chaemical Co., Ltd., respectively. All solvents were distilled prior to use.

\subsection{Instruments and Equipments}

T22S Visible spectrophotometer (Chengdu BSIDA Instrument Co.Ltd.); HHS electro thermal constant temperature water bath kettle (Shanghai Leiyun Test Instrument manufacturing Co.Ltd.); JY92-2D ultrasonic cell grinder (Nanjing Pusen instrument Co.Ltd.); SHB-3 rotary evaporator (Shanghai Dibo Laboratory Equipment Co.Ltd.); T6-New century ultraviolet visible spectrophotometer (Beijing Puxi Tongyong instrument Co.Ltd.); TDL-40B-W desktop low speed larger capacity centrifuge (Shanghai Anting Scientific instrument factory); ALPHAL-2 Ireeze drier (Xianan BILON biotechnology Co.Ltd.) were employed in this research

\subsection{Extraction Procedure}

L.lucidus $\rightarrow$ Smash $\rightarrow$ Ultrasonication $\rightarrow$ The petroleum ether degreasing $\rightarrow$ Deproteinization $\rightarrow$ Decoloration $\rightarrow$ Dialysis $\rightarrow$ Concentration $\rightarrow$ Freeze drying $\rightarrow$ Determination of total Lycopus lucidus Tur@2. polysaccharide content.

\subsection{Making the Glucose Standard Curve}

Take method of Phenol-sulfuric $\{3\}$

\subsection{The Measure of Total Reductive Sugar Content}

Taking the measure of following reference [2]: adding $1.0 \mathrm{~mL}$ extract and $99.0 \mathrm{~mL}$ distilled water into a $100 \mathrm{~mL}$ of volumetric flask. Sucking $2.0 \mathrm{~mL}$ of this dilute solution to a test tube, then putting into $1.0 \mathrm{~mL} 6 \%$ mass fraction of phenol, as well as $5.0 \mathrm{~mL} 98 \%$ mass fraction of concentrated sulfuric acid respectively, finally keeping them at room temperature for 20 mins. Calculating its polysaccharide content and extraction yield according to the following formula.

LTP yield $\%=$ total saccharide content $(\mathrm{g}) /$ material dry weight $(\mathrm{g}) \times 100$

\subsection{The Single Factor Test of Crude Polysaccharide}

Do research on the effect of different ratio of water to material on LIP yield under the condition of $200 \mathrm{~W}$ of supersonic power, 30mins of acting time; the second is research on the effect of different supersonic power on LIP yield under the condition of 40:1 or water to material, $30 \mathrm{mins}$ of acting time; the third is research on the ef fect of different supersonic action time on LIP yield under the condition of 40:1 of water to material, $200 \mathrm{~W}$ of supersonic power. Each test repeated three times and data were analyzed statistically by SPSS15.0 software [3].

\subsection{The Design of Optimization Experiment of the Extraction of Polysaccharide by Response Surface Methodology}

Based on the single factor experiment, determining the Box-Behnken design variables, taking LTP yield as response value, the extraction conditions were optimized by the response surface analysis. 


\subsection{The Antibacterial Experiment of LTP}

Activating the bacterial spawn mentioned above in the appropriate slant culturemedium. The material of positive control experiment is ampicillin. Making its concentration about 103 104 CFU/mL bacteria liquid respectively as reserve with sterile saline. Taking a strong hydroscopic filter paper and making it a round one with a $12 \mathrm{~mm}$ of diameter. Soaking them in the sterile LTP solution for $24 \mathrm{~h}$ after sterilization. Drying them at the temperature of $37^{\circ} \mathrm{C}$ as reserve.

Absorbing bacteria liquid $0.2 \mathrm{~mL}$ to make it into bacterial plate. According to aseptic operating requirements, dried filter paper containing LTP place the corresponding position of medium surface. Each plate put 4 pieces, 3 of which as samples, 1 of which is sterile physiological saline as comparison.

Culturing them in the constant incubator $\left(36 \sim 37^{\circ} \mathrm{C}, 18 \sim 24 \mathrm{~h}\right)$. Taking them out and measuring the diameter of antibacterial zone. And then comparing antibacterials activity finally.

\section{Result and Discussion}

\subsection{Making Standard Curve of Glucose Solution}

Taking the method of reference [2], absorbance was determined. Taking the absorbance as ordinate and glucose as the abscissa, regression was performed. The result was as followed: $\mathrm{Y}=0.0126 \mathrm{X}-0.0017, \mathrm{R}^{2}=0.9999$

\subsection{Effect of Ratio of Water to Material on LTP Yield}

With the increase of ratio of warer to material, the LTP yield showed a tendency of ascending. The fact that increasing solvent quantity contributed greatly to the LTP diffusion can explain However, ratio of water to material was more than 40:1, the increasing rate of LTP yield slowed down and became moderate gradually, which indicated that the exchange between solid and liquid reached balance fundamentally.

At this time, almost all of the LTP have dissolved out. Continuing to increase ratio of water to material will cause a waste of solvent and it is also not easy to the recycling. So 40.1 is just the appropriate ratio.

\subsection{Effect of Supersonic Power on LTP Yield}

The result indjeated that when the supersonic power increased within the range of $500 \mathrm{~W}$, the LTP xield also kept rising. When enlarging the supersonic power, the speed of solvent moved faster, and the L.lucidus have completely swollen out. Consequentry, more and more solvent entered the cells which caused the materials inside cell easier permeating out. The LTP yield indicated the trend of declination when the power was beyond the range of $500 \mathrm{~W}$. That's probably the increasing power generated higher temperature, the LTP began to degenerate, and the LTP yield was influenced by that eventually. So $500 \mathrm{~W}$ was the appropriate power.

\subsection{Effect of Supersonic Time on LTP Yield}

The cavitations and concussion of supersonic help diffuse material inside cells. Under the condition of 40:1 of ratio of water to material and 500W of supersonic power, the LTP yield increased continually when extending acting time, which increased obviously in 50mins, and tended to moderate afterwards. Considering extraction time, energy consumption and the practical effect, 50mins was the appropriate acting time. 
Optimizing the technological conditions of ultrasonic-assisted extraction of polysaccharide from $L$.lucidus by response surface methodology

According to the Box-Benhnken center-united experimental design principles and the result of single factor experiment, take ratio of water to material $\left(X_{1}\right)$, supersonic power $\left(X_{2}\right)$, supersonic acting time $\left(X_{3}\right)$ as independent variables, and the LTP yield as response value. Table 1 showed the experiment factors and level design, Table 2 showed experiment result.

Table 1. Factors and Level Value of Response Surface Analysis

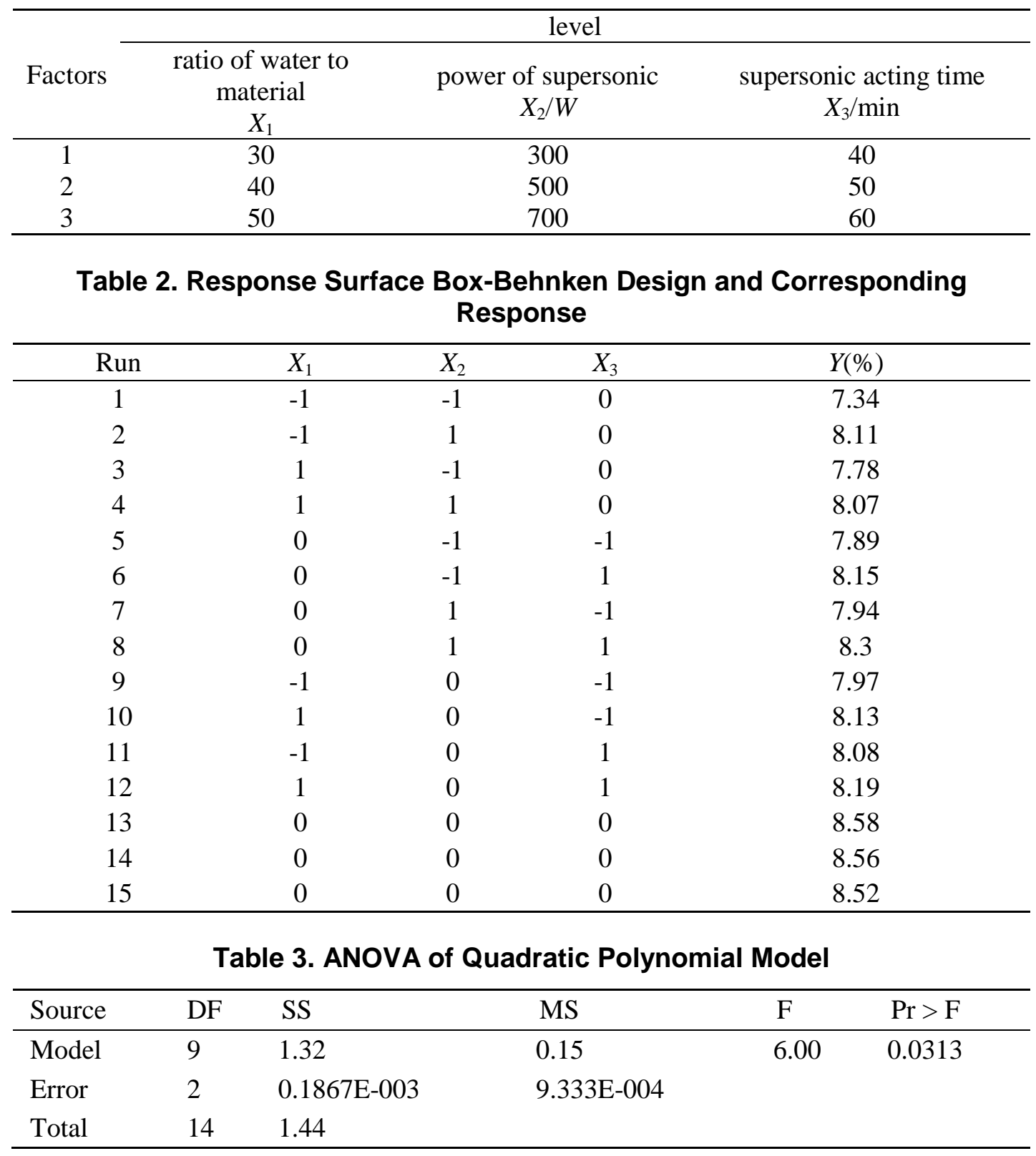


Table 4. Significance Test of Regression Coefficient

\begin{tabular}{cccc}
\hline Term & Estimate & $t$ & $\operatorname{Pr}>|t|$ \\
\hline$X_{1}$ & 0.08375 & 0.1895 & 0.00076 \\
$X_{2}$ & 0.1575 & 0.0356 & 0.04210 \\
$X_{3}$ & 0.09875 & 0.1335 & 0.05244 \\
$X_{1} X_{1}$ & -0.12 & 0.1846 & 0.00000 \\
$X_{1} X_{2}$ & -0.0125 & 0.8790 & 0.00000 \\
$X_{1} X_{3}$ & 0.025 & 0.7616 & 0.00487 \\
$X_{2} X_{2}$ & -0.35292 & 0.0074 & 0.00639 \\
$X_{2} X_{3}$ & -0.37542 & 0.0057 & 0.15148 \\
$X_{3} X_{3}$ & -0.10792 & 0.2413 & 0.68994 \\
\hline
\end{tabular}

Table 5. Bacteriostatic Activity of LTP

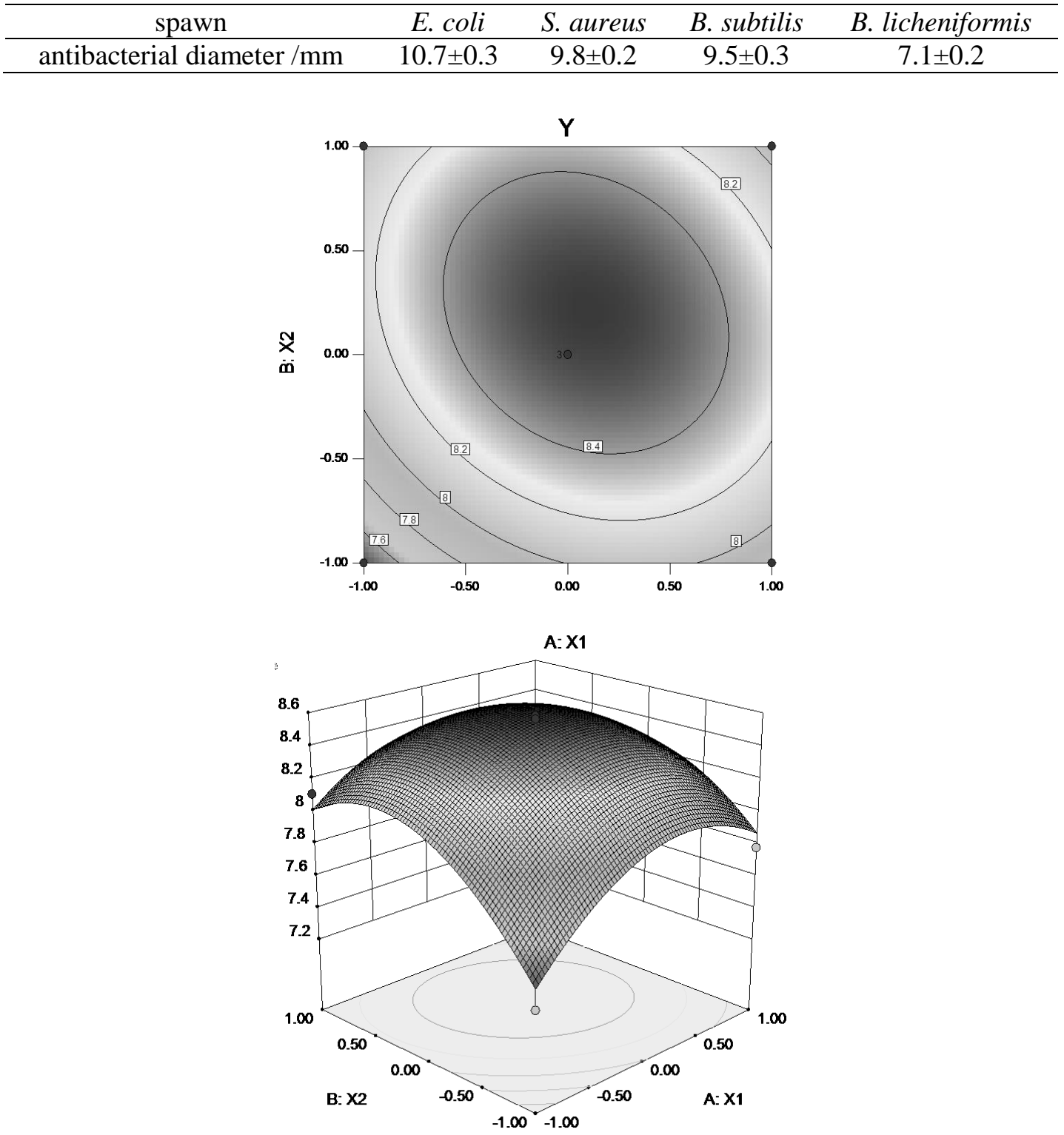

Figure 1. Responsive Surfaces and Contours of $Y=F(X 1, X 2)$ 

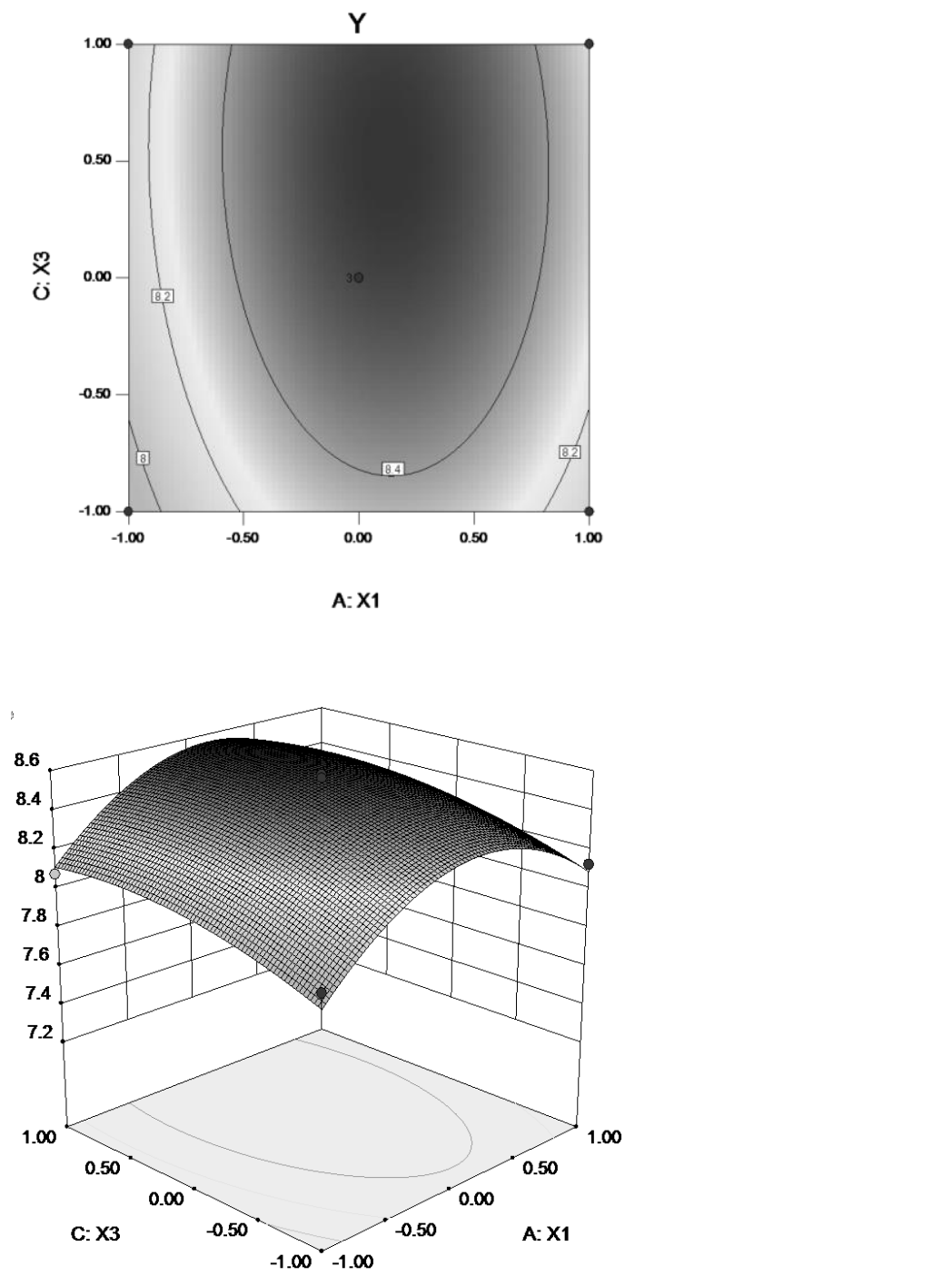

Figure 2. Responsive Surfaces and Contours of $Y=F(X 1, X 3)$
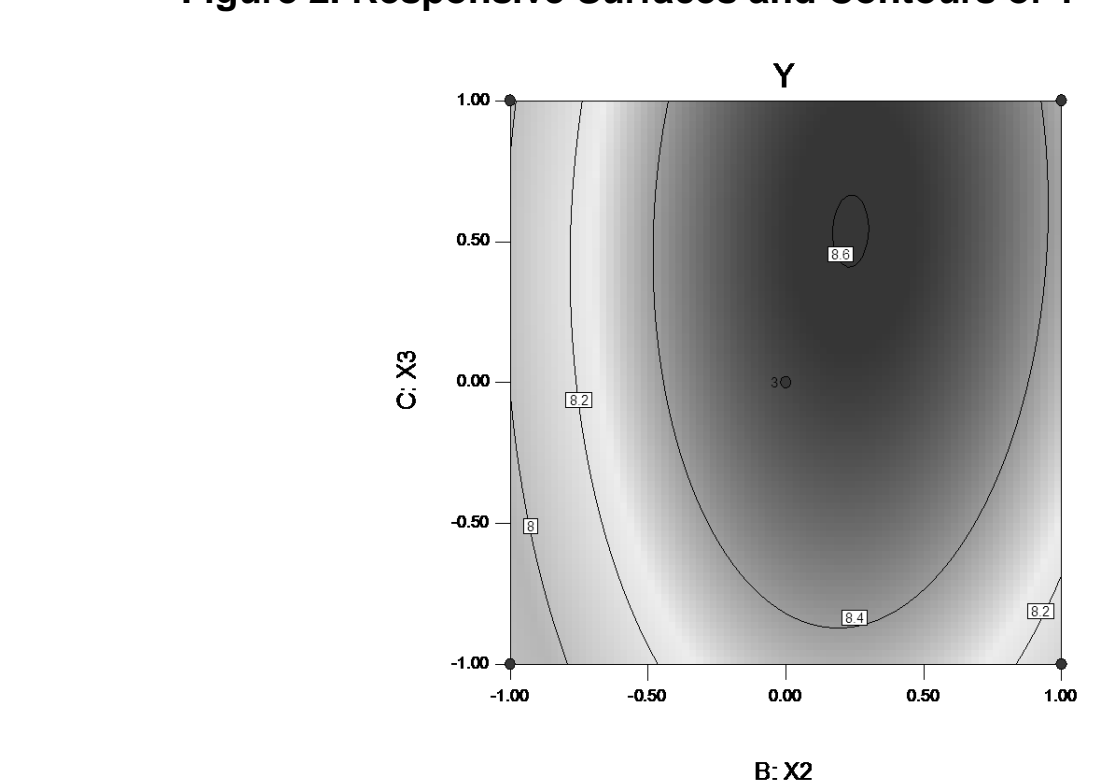


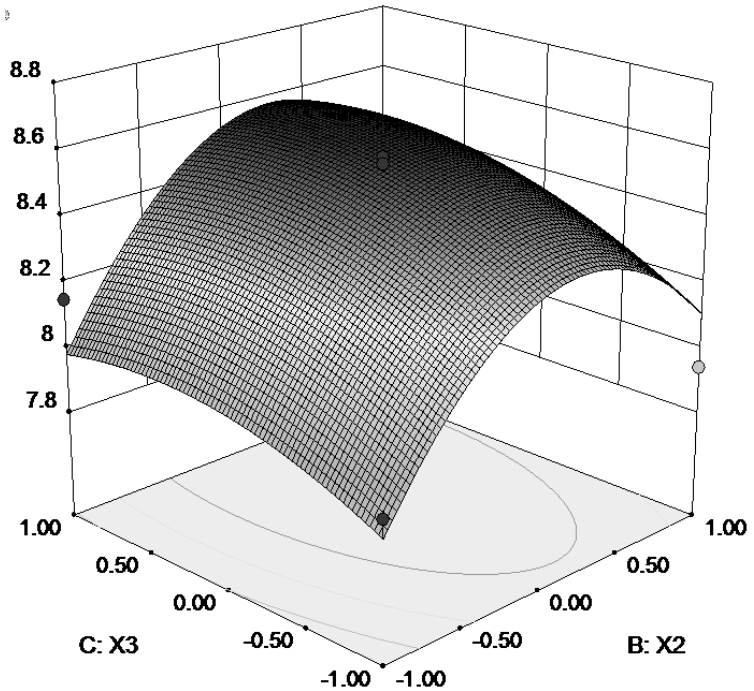

Figure 3. Responsive Surfaces and Contours of $Y=F(X 2, X 3)$

\section{Result of Response Surface Analysis}

The third factor of center-untied experimental needed 15 times in all. A multiple quadratic equation was fitted, which aimed to describe experimental model of dependent variable and independent variables. A multiple regression equation was fitted by the SAS 9.2 software.

$$
\begin{gathered}
Y=8.55+0.0840 \times X_{1}+016 \times X_{2}+0.099 \times X_{3}-0.12 \times X_{1} \times X_{2}-0.013 \times X_{1} \times X_{3}+0.025 \times X_{2} \times X_{3}- \\
0.35 \times X_{12}-0.38 \times X_{25}-0.11 \times X_{32}
\end{gathered}
$$

The result showed that the linear correlation was $R_{2}=0.9568$ and the fitting degree of regression equation was high. The optimal value $X_{1}=0.08 、 X_{2}=0.2 、 X_{3}=0.42$ were obtained by the SAS 9.2 software. Namely, the best extraction condition of LTP is: ratio of water to materral $40.73,542.70 \mathrm{~W}$ of supersonic, acting time 54.78 min, under which condition on $8.59 \%$ predicted value of LTP yield could be attained.

Responsive surfaces and contours were made out according to the response surface analysis and the regression equation. Figure 1-3 showed the details.

The table 4 showed that the order of influencial factor on LTP yield in turn is ratio of water to materials $>$ power of supersonic $>$ acting time. According to kyrtograph of the three dimensional space structured by the factors of $X_{1}, X_{2}, X_{3}$ and the response value from Figure 1-3, influence of three factors $\left(X_{1}, X_{2}, X_{3}\right)$ on response valuecould be reflected out.

The esponse surface plot structured by the ratio of water to material and power of supersonic was much steeper than these two ones structured by the ratio of water to material and acting time, acting time and power of supersonic respectively. Meantime, the response value circle of contour was more intensive, and the interaction showed apparently most. In order to verify the accuracy of experimental model prediction, verification tests on the above optimized conditions were carried out. After 5 times duplicate tests and calculating the average value afterwards, the practical LTP yield was $8.55 \%$, approaching the model prediction value with just $0.04 \%$ of error, which denoted that the model could predict practice well and provide further references for the production of LTP. Cheng Guiyuan et al. also studied the extraction process of Polysaccharides from L.lucidus with good 
consequences [4]. In his study, Temperature was set as one of the parameters, and the best extraction temperature was $80^{\circ} \mathrm{C}$. In this study, Extraction process was carried out at the room temperature, lessening the detriment to the other nutrients of raw material.

Taking E.coli, S.aureus, B.subtilis, and B.licheniformis as experimental spawn, the LTP antibacterial activity was studied by filtering paper method. We can know from reference [5], the LTP had no or inconspicuous antibacterial activity when the diameter of antibacterial zone was less than $6.5 \mathrm{~mm}$; the LTP showed antibacterial activity when $6.5 \mathrm{~mm} \leq \mathrm{r}<8 \mathrm{~mm}$; its antibacterial activity was obvious when 8.0 $\mathrm{mm} \leq \mathrm{r}<10.0 \mathrm{~mm}$; its antibacterial activity was pretty obvious when $\mathrm{r} \geq 10.0 \mathrm{~mm}$. Table 5 showed the result of experiment: the average antibacterial diameter of LTP on E.coli, B.subtilis, S.aureus reached $10.7 \mathrm{~mm}, 9.8 \mathrm{~mm}$ and $9.5 \mathrm{~mm}$ respectively, which meaned these three bacteria had much strong antibacterial activity. However, the average antibacterial diameter of LTP on the B.licheniformis reached $7.1 \mathrm{~mm}$, which indicated the LTP has obvious generally antibacterial activity on it.

\section{Conclusion}

The LTP extraction process was optimized by Box-Benhnken experiment of response surface methodology. Result showed that the order of influencial factor on LTP yield in turn is: the ratio of water to material > powe of supersonic > supersonic acting time. Under the conditions of 40.73 of the ratio of water to material, $542.70 \mathrm{~W}$ of power of superson c and $54.78 \mathrm{cmins}$ of supersonic acting time, as much as $8.59 \%$ of the LTP could be obtained, closing to the calculated value of $8.55 \%$. The data fitted well which meaned the regression equation could predict the extraction effect of the ETPaccurately.

The LTP antibacterial activity was studied by filtering paper method. The result showed that the LTP had strong antibacterial activity on E.coli, B.subtilis, S.aureus, and its average antibacteria diameter could reach $10.7 \mathrm{~mm}, 9.8 \mathrm{~mm}, 9.5 \mathrm{~mm}$ respectively, but the average antibacterial diameter of the B.licheniformis reached $7.1 \mathrm{~mm}$ which meaned has omparatively obvious antibacterial activity.

\section{Acknowledgements}

This work is supported by 2014 Educational Commission of Anhui Province of China (NO.KJ2014A243) and 2016 The Key Program in the Youth Elite Support Plan in Universitie Ar Anhui Province (NO.gxyqZD2016304).

\section{References}

[1] J. Cui, Yue, F. Tang and J. Wang, "HPTLC analysis of the flavonoids in eight species of indocalamus leaves", Jpc-J Planar Chromatography, vol.24, (2011), pp. 394-399

[2] J.M Scurlock, D.C. Dayton and B. Hames, "Bamboo: an overlooked biomass resource?" biomass \& bioenergy, vol. 19, no.4, (2000), pp. 229-244.

[3] H.Q. Cao, Y.D. Yue and Z.H. Peng, "Evaluation of extracts from bamboo for biological activity against mosquitoes", Entomologia Sinica. vol. 11, (2004), pp. 267-273.

[4] G. Chen, C. Zhang and Y. Luo, "Extraction of Polysaccharides from Lycopus lucidus", Chinese Journal of Experimental Traditional Medical Formulae. vol. 17, no. 21, (2011, pp.38-40).

[5] J. Jiao, Y. Zhang and C. Liu, "Separation and purification of tricin from an antioxidant product derived from bamboo leaves", J Agric Food Chem., vol. 55, no.25, (2007), pp. 10086-10092. 


\section{Author}

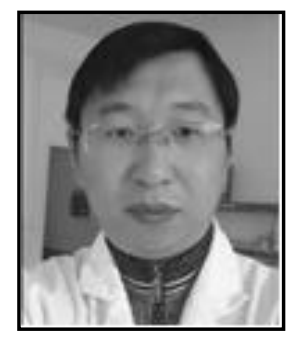

Shenglang Jin, he was born on April 2, 1980, in Huangshan, China.

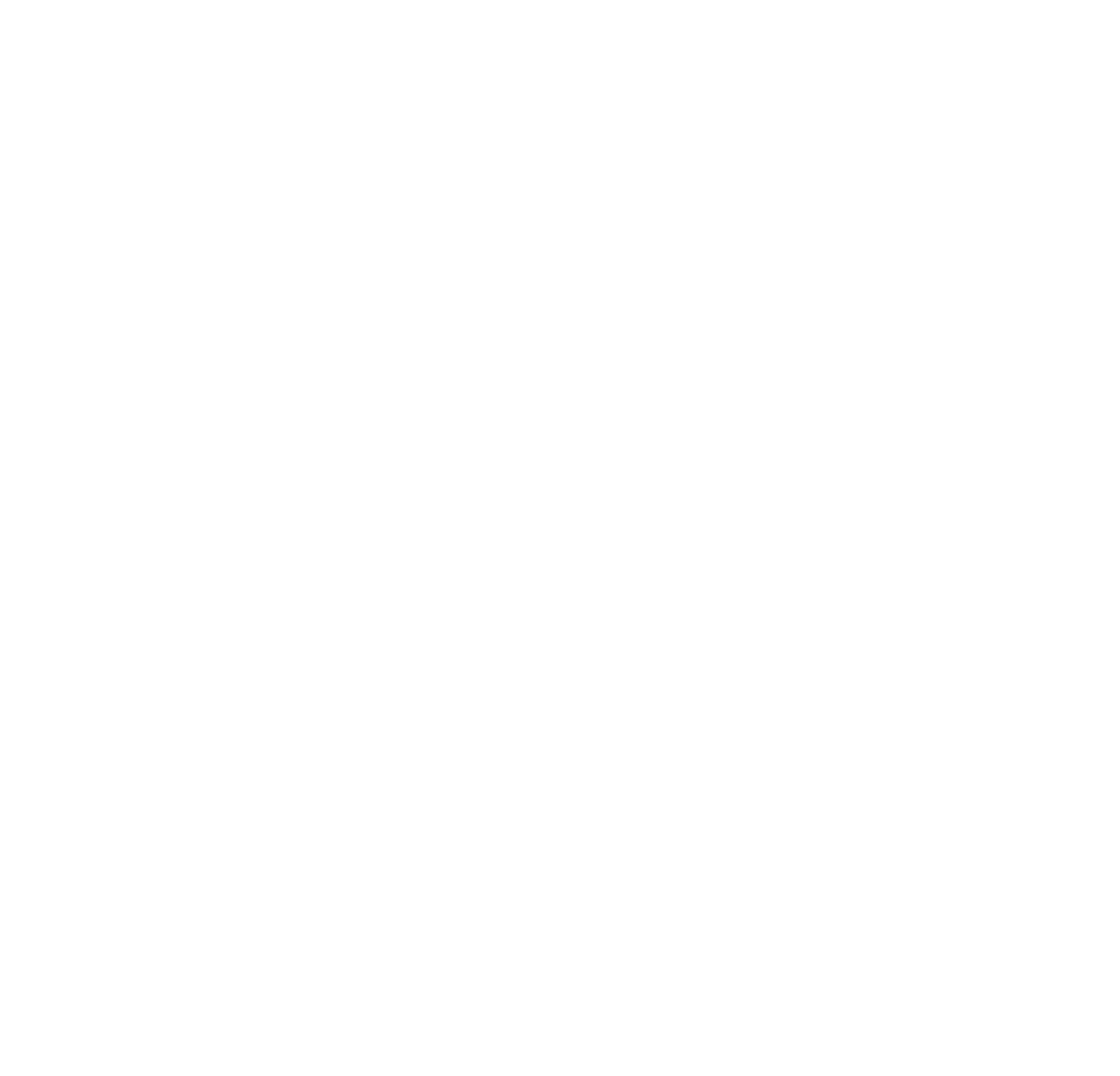


International Journal of Hybrid Information Technology

Vol. 9, No.11 (2016)

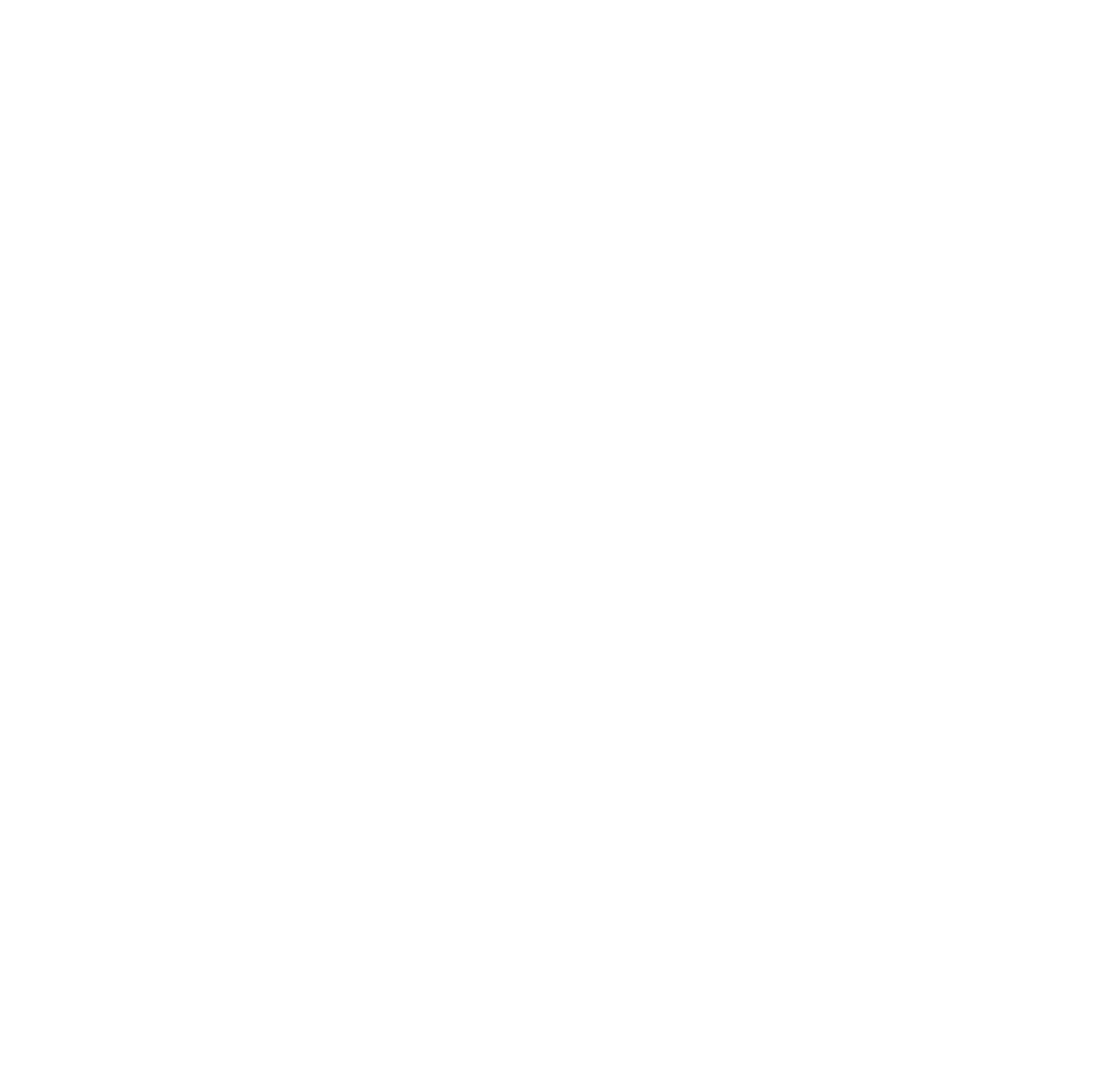

